\title{
The energetic particle intensity estimated from cosmogenic isotope Al-26 produced in lunar samples
}

\section{Stepan Poluianov*}

Space Climate Research Unit, University of Oulu, Finland,

Sodankylä Geophysical Observatory, University of Oulu, Finland,

E-mail: stepan.poluianov@oulu.fi

\section{Gennady Kovaltsov}

Ioffe Physical-Technical Institute, St. Petersburg, Russia.

\section{Ilya Usoskin}

Space Climate Research Unit, University of Oulu, Finland,

Sodankylä Geophysical Observatory, University of Oulu, Finland.

\begin{abstract}
Direct measurements of solar energetic particles (SEP) became possible only in the space era covering past several decades. However, for many academic and practical reasons, it is important to know the SEP energy spectrum on much longer time scales in the past. Such information can be obtained using reconstructions based on cosmogenic radioisotope measurements in extra-terrestrial objects without magnetic and atmospheric shielding such as lunar surface or meteoroids. Thanks to the Apollo missions, samples of lunar rocks have been brought to the Earth and measured for the isotope content. Although estimates of the average SEP energy spectrum from cosmogenic radionuclides measured in lunar samples have been made earlier, here we revisit the approach using newly calculated depth profile of the yield function for ${ }^{26} \mathrm{Al}$ in lunar rocks. We have developed a full Monte-Carlo model of the nuclide production by energetic particles in the rock. As a result, we present the improved estimate of the average solar energetic particle intensity at $1 \mathrm{AU}$ on the multi-millenial time scales.
\end{abstract}

35th International Cosmic Ray Conference - ICRC2017

10-20 July, 2017

Bexco, Busan, Korea

${ }^{*}$ Speaker. 


\section{Introduction}

The only known method to study solar energetic particles (SEP) on the long time scales (thousands to millions years before modern measurements) is based on cosmogenic nuclides produced in extraterrestrial bodies such as meteoroids and the Moon. Those objects are not protected by magnetic field and/or atmosphere, and thus relatively low-energy SEP can hit their surface and leave a footprint in the form of produced isotopes. Thanks to Apollo missions, several lunar samples have been brought to the Earth and measured for the nuclide content and its depth distribution. Estimates of the energetic particle intensity have been conducted earlier [e.g., 1, 2, 3, 4] via quantitative modelling of nuclide production by galactic cosmic rays (GCR) and SEP. Since most of results were obtained decades ago and the quality of modelling of the cosmic ray cascade has increased significantly, the study of cosmogenic nuclide need a revision. Moreover, earlier works considered production of nuclides only by secondary protons, neutrons and $\alpha$-particles, though Li et al. [5] have shown that the contribution by secondary charged pions cannot be neglected.

Because of that, we revised the estimate of GCR and SEP energy spectra with a newly calculated detailed depth profile of the isotope ${ }^{26} \mathrm{Al}$ (half-life $7.05 \cdot 10^{5}$ years) yield function in a lunar sample including production by secondary pions.

\section{Model of the isotope production}

We used the standard yield-function approach [e.g., 6, 7] for quantitative modelling of the isotope production rate $Q(h)$ in a lunar sample:

$$
Q(h)=\sum_{i} \int_{0}^{\infty} Y_{i}(h, E) J_{i}(E) d E
$$

where $h$ is the depth (in $\mathrm{g} / \mathrm{cm}^{2}$ ), $i$ is the index of primary particles' type (e.g., protons, $\alpha$-particles), $Y_{i}(h, E)$ is the isotope's yield function (in atoms $\mathrm{cm}^{2} \mathrm{sr} / \mathrm{g}$ ), $J_{i}$ is the differential intensity of $i$ th primary particles (in $\left(\mathrm{cm}^{2} \mathrm{~s} \mathrm{sr} \mathrm{MeV}\right)^{-1}$ ), and $E$ is the energy. The units of $Q(h)$ are atoms $\mathrm{g}^{-1} \mathrm{~s}^{-1}$.

The method significantly simplifies computation of the production rate if the yield function is known. In our case we calculated new ${ }^{26} \mathrm{Al}$ yield functions with high energy and depth resolution. We considered production of the isotope by primary protons and $\alpha$-particles, assuming that the contribution from heavier species can be estimated by scaling of the production by $\alpha$-particles [6, 7]. The computation required simulation of the nuclear cascade, which was done by a Monte Carlo algorithm implemented with the toolkit GEANT4.10.0 [8,9]. We designed a realistically sized thick flat lunar sample according to the composition adopted from [10]. Its surface was simulated to be bombarded by monoenergetic primary particles with the isotropic angular distribution. The grid of initial particle energies was set as quasilogarithmic from 0.005 to $100 \mathrm{GeV} /$ nuc (with 5-10 points per decade). We "registered" cascade protons, neutrons, $\alpha$-particles, pions-plus and pionsminus crossing the depth layers from 0 to $950 \mathrm{~g} / \mathrm{cm}^{2}$ distributed with quasilogarithmic steps from 0.01 to $50 \mathrm{~g} / \mathrm{cm}^{2}$ from top to bottom. We included production of ${ }^{26} \mathrm{Al}$ by pions because their contribution is not negligible in dense matter, as noted by Li et al. [5]. The cross-sections of pion reactions were obtained by direct simulations with GEANT4. The method of computation of the 
yield function using a Monte Carlo simulation of the cosmic ray cascade is described in detail in, e.g., [11].

Equation 2.1 requires the differential intensity of primary energetic particles $J(E)$ along with the yield function $Y(h, E)$. For galactic cosmic rays, we used the force-field approach [e.g., 12] with the local interstellar spectrum from [13] and modulation potential $\phi$ representing the heliospheric modulation of GCR.

The differential intensity $J(R)$ (in $\left(\mathrm{cm}^{2} \mathrm{~s} \mathrm{MV}\right)^{-1}$ ) of solar energetic particles is often represented by an exponential function of the magnetic rigidity $R$ [1]:

$$
J(R)=J_{0} \exp \left(-\frac{R}{R_{0}}\right),
$$

where $J_{0}$ and $R_{0}$ are free parameters in units $\left(\mathrm{cm}^{2} \mathrm{~s} \mathrm{MV}\right)^{-1}$ and $\mathrm{MV}$, respectively.

We computed the ${ }^{26} \mathrm{Al}$ production rates for different GCR and SEP spectra covering the realistic ranges of parameters $\phi, J_{0}$ and $R_{0}$ for further fitting into the measured data of the isotope content described below.

\section{Fitting the measurements and model}

The results of our production model were compared with the measured concentration of ${ }^{26} \mathrm{Al}$ in different lunar rocks and cores brought during Apollo missions [3, 4, 14, 15]. The data are plotted in Figure 1 as color dots with error bars.

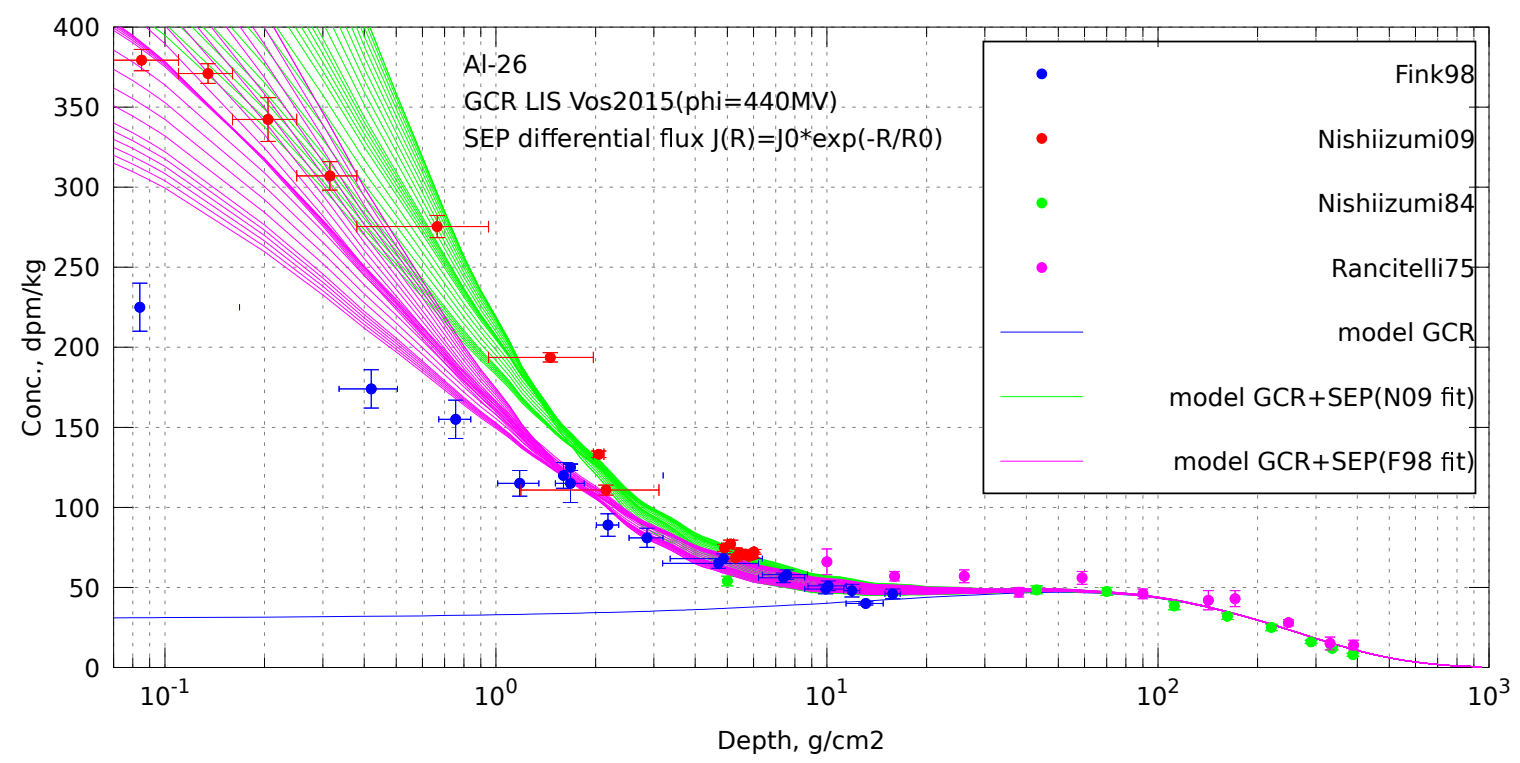

Figure 1: Content of ${ }^{26} \mathrm{Al}$ in lunar samples. Measurements are denoted as dots with error bars (see the legend for particular references). Modelled production of ${ }^{26} \mathrm{Al}$ by GCR (the modulation potential $\phi=440$ MV) is denoted by the blue curve, modelled production by SEP are indicated by green and magenta curves (fitting into the data from [4] and [3], respectively). Labels Vos2015, Fink98, Nishiizumi09, Nishiizumi84, Rancitelli75 correspond to [13], [3], [4], [15], and [14], respectively.

The criterion of minimization of $\chi^{2}$ was used in all fitting procedures in this work. Assuming that at the depths $\geq 20 \mathrm{~g} / \mathrm{cm}^{2}$ production of ${ }^{26} \mathrm{Al}$ is driven mostly by GCR, we fitted the modelled 
curve into the joint data set from [14] and [15] (Figure 1, the blue curve). It gave us the best fit modulation potential $\phi=440 \mathrm{MV}$ (for the local interstellar spectrum from [13]). It represents the average level of solar activity for the past several half-life times of ${ }^{26} \mathrm{Al}$ (the timescale of a million years) that is slightly below the modern average level covering the past 6 solar cycles.

The contribution of SEP is the remainder after subtracting the modelled curve of GCR-produced ${ }^{26} \mathrm{Al}$ from the measured data at $<20 \mathrm{~g} / \mathrm{cm}^{2}$. Because of notable difference between the concentrations from [3] and [4], we conducted two fitting procedures for each data set varying the spectral parameters $J_{0}$ and $R_{0}$. The distributions of the criterion $\chi^{2}\left(R_{0}, J_{0}\right)$ are shown in Figure 2. One can see that, although the absolute minimum of $\chi^{2}$ can be defined, there is a wide range of parameters $R_{0}$ and $J_{0}$ (denoted by black curves in Figure 2) providing quality of the fit close to the best. This shows that it is hardly possible to reconstruct unambiguous average spectrum of SEP according to the exponential model (Equation 2.2) from only ${ }^{26} \mathrm{Al}$ data without additional assumptions. However, the family of SEP energy spectra can be derived from the pairs of $R_{0}$ and $J_{0}$ related to low $\chi^{2}$ (Figure 3). The ${ }^{26} \mathrm{Al}$ production rates corresponding to these spectra are shown in Figure 1 as green and magenta curves.
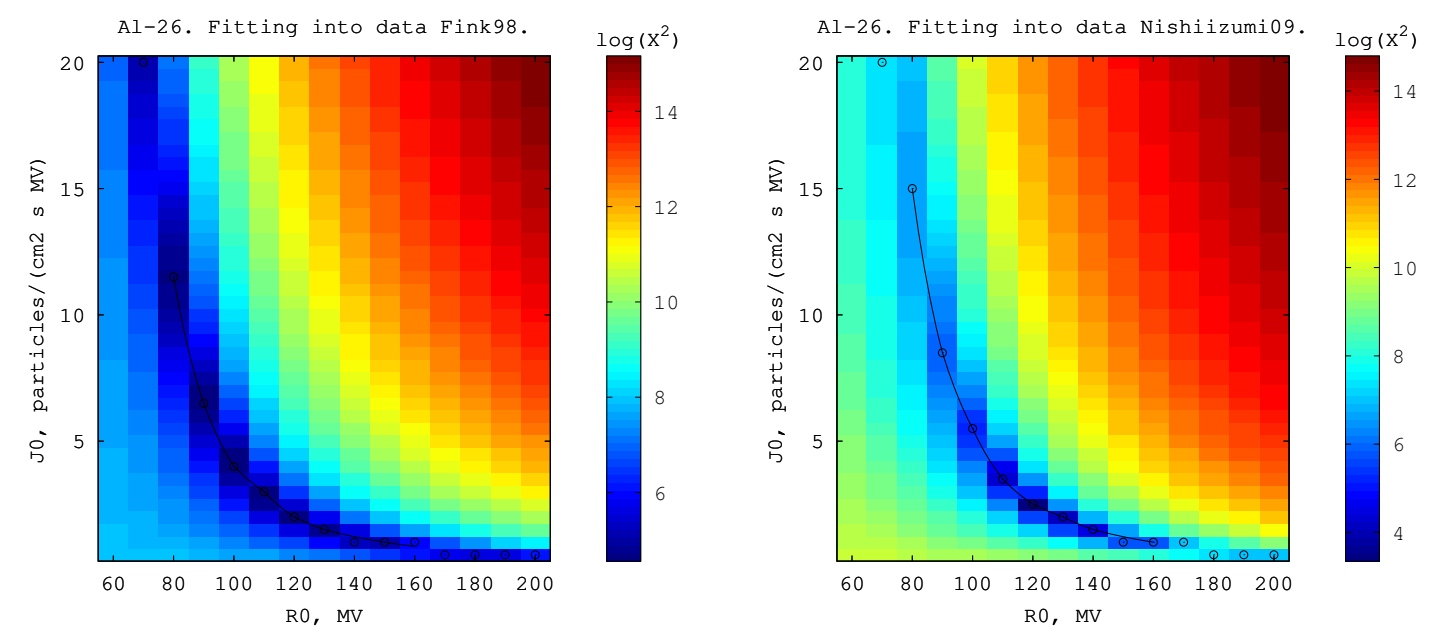

Figure 2: Distributions of the criterion $\chi^{2}\left(R_{0}, J_{0}\right)$ as a function of SEP spectral parameters for fitting the modelled $\operatorname{GCR}(\phi=440 \mathrm{MV})+\mathrm{SEP}$ production rate of ${ }^{26} \mathrm{Al}$. The left and right panels show fitting into the data from [3] and [4], respectively. The black curves indicate parameters $R_{0}$ and $J_{0}$ close to the best fit.

\section{Summary}

In this work we calculated the yield functions of ${ }^{26} \mathrm{Al}$ produced by energetic protons and $\alpha$ particles in a lunar sample. The results take also the production by pions into account, as noted by [5]. Using the obtained yield functions, we computed the ${ }^{26} \mathrm{Al}$ production rates by galactic cosmic rays and solar energetic particles as functions of corresponding spectral parameters. The results were fitted into experimental data with the optimal value of the heliospheric modulation potential $\phi=440 \mathrm{MV}$ (the local interstellar spectrum from [13]) averaged during last several hundred thousand years. Fitting the SEP production rates into data from [3] and [4] has shown that it is hardly possible to reconstruct unambiguous energy spectrum of solar particles. Instead of that, families of 

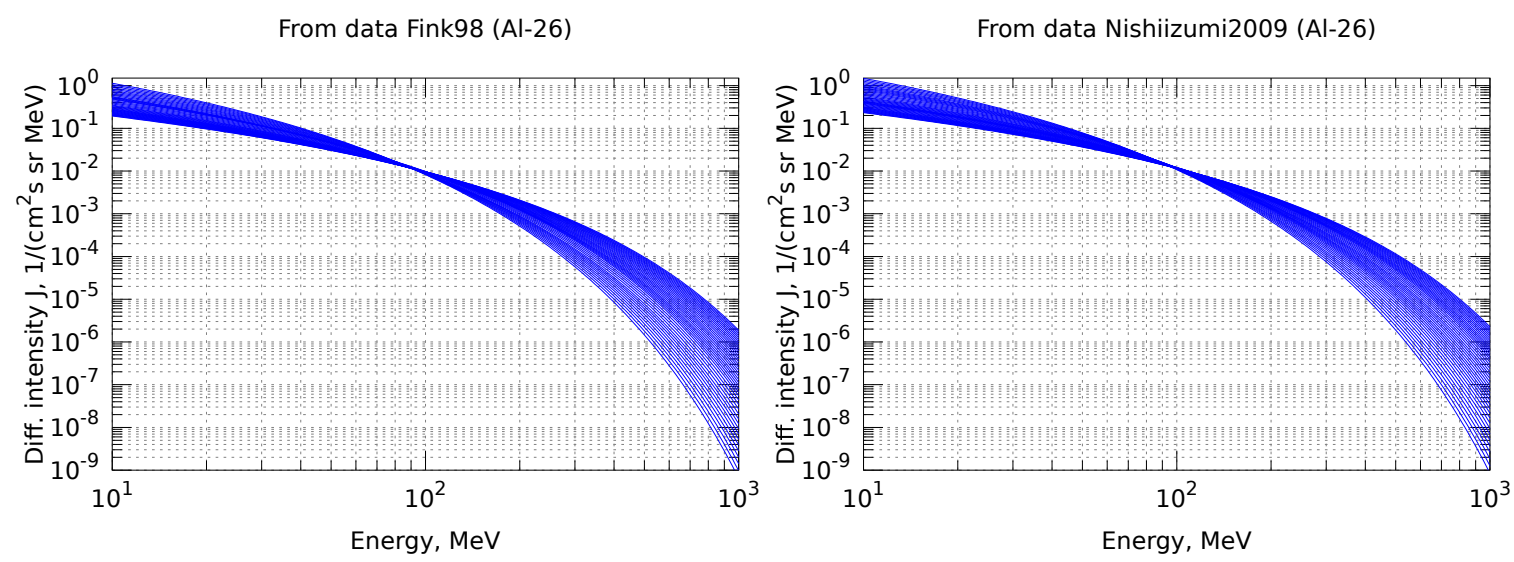

Figure 3: Differential intensities of SEP derived from families of the best fit parameters $R_{0}$ and $J_{0}$. The left and right panels correspond to fitting modelled ${ }^{26} \mathrm{Al}$ production rates into the data from [3] and [4], respectively.

the SEP differential intensities $J(E)$, which fit the isotope data approximately equally well, were obtained and shown in Figure 3.

\section{References}

[1] R. C. Reedy and J. R. Arnold. Interaction of solar and galactic cosmic-ray particles with the Moon. J. Geophys. Res., 77:537, 1972. doi: 10.1029/JA077i004p00537.

[2] G. P. Russ, III and M. T. Emerson. Mn-53 and Al-26 evidence for solar cosmic ray constancy - an improved model for interpretation. In R. O. Pepin, J. A. Eddy, and R. B. Merrill, editors, The Ancient Sun: Fossil Record in the Earth, Moon and Meteorites, pages 387-399, 1980.

[3] D. Fink, J. Klein, R. Middleton, S. Vogt, G. F. Herzog, and R. C. Reedy. ${ }^{41} \mathrm{Ca},{ }^{26} \mathrm{Al}$, and ${ }^{10} \mathrm{Be}$ in lunar basalt 74275 and ${ }^{10} \mathrm{Be}$ in the double drive tube $74002 / 74001$. Geochimica et Cosmochimica Acta, 62:2389-2402, July 1998. doi: 10.1016/S0016-7037(98)00134-3.

[4] K. Nishiizumi, J.R. Arnold, C.P. Kohl, M.W. Caffee, J. Masarik, and R.C. Reedy. Solar cosmic ray records in lunar rock 64455. Geochimica et Cosmochimica Acta, 73(7): 2163-2176, 2009. ISSN 0016-7037. doi: http://dx.doi.org/10.1016/j.gca.2008.12.021. URL http://www.sciencedirect.com/science/article/pii/s0016703709000143.

[5] Y. Li, X. Zhang, W. Dong, Z. Ren, T. Dong, and A. Xu. Simulation of the production rates of cosmogenic nuclides on the Moon based on Geant4. J. Geophys. Res. (Space Phys.), 122: 1473-1486, February 2017. doi: 10.1002/2016JA023308.

[6] W.R. Webber and P.R. Higbie. Production of cosmogenic be nuclei in the earth's atmosphere by cosmic rays: Its dependence on solar modulation and the interstellar cosmic ray spectrum. J. Geophys. Res., 108:1355, 2003. doi: 10.1029/2003JA009863. 
[7] G. A. Kovaltsov and I. G. Usoskin. A new 3D numerical model of cosmogenic nuclide ${ }^{10}$ Be production in the atmosphere. Earth Planet. Sci. Lett., 291:182-188, March 2010. doi: 10.1016/j.eps1.2010.01.011.

[8] S. Agostinelli, J. Allison, K. Amako, et al. Geant4 - a simulation toolkit. Nucl. Instr. Meth. Phys. A, 506(3):250-303, 2003. ISSN 0168-9002. doi: http://dx.doi.org/10.1016/S0168-9002(03)01368-8. URL http://www.sciencedirect.com/science/article/pii/s0168900203013688.

[9] J. Allison, K. Amako, J. Apostolakis, et al. Geant4 developments and applications. Nuclear Science, IEEE Transactions on, 53(1):270-278, Feb 2006. ISSN 0018-9499. doi: 10.1109/TNS.2006.869826.

[10] R. C. Finkel, J. R. Arnold, M. Imamura, R. C. Reedy, J. S. Fruchter, H. H. Loosli, J. C. Evans, A. C. Delany, and J. P. Shedlovsky. Depth variation of cosmogenic nuclides in a lunar surface rock and lunar soil. In Lunar Planet. Sci. Conf. Proceedings, volume 2 of Lunar and Planetary Science Conference Proceedings, page 1773, 1971.

[11] S. V. Poluianov, G. A. Kovaltsov, A. L. Mishev, and I. G. Usoskin. Production of cosmogenic isotopes ${ }^{7} \mathrm{Be},{ }^{10} \mathrm{Be},{ }^{14} \mathrm{C},{ }^{22} \mathrm{Na}$, and ${ }^{36} \mathrm{Cl}$ in the atmosphere: Altitudinal profiles of yield functions. Journal of Geophysical Research (Atmospheres), 121:8125-8136, July 2016. doi: 10.1002/2016JD025034.

[12] K. Herbst, A. Kopp, B. Heber, F. Steinhilber, H. Fichtner, K. Scherer, and D. MatthiÃd'. On the importance of the local interstellar spectrum for the solar modulation parameter. J. Geophys. Res.: Atmos., 115(D1):D00I20, 2010. ISSN 2156-2202. doi: 10.1029/2009JD012557. URL http://dx.doi.org/10.1029/2009JD012557.

[13] E. E. Vos and M. S. Potgieter. New Modeling of Galactic Proton Modulation during the Minimum of Solar Cycle 23/24. Astrophys. J., 815:119, December 2015. doi: 10.1088/0004$637 X / 815 / 2 / 119$.

[14] L. A. Rancitelli, J. S. Fruchter, W. D. Felix, R. W. Perkins, and N. A. Wogman. Cosmogenic isotope production in Apollo deep-core samples. In Lunar and Planetary Science Conference Proceedings, volume 6 of Lunar and Planetary Science Conference Proceedings, pages 1891-1899, 1975.

[15] K. Nishiizumi, J. R. Arnold, J. Klein, and R. Middleton. Al-26 depth profile in Apollo 15 drill core. Earth Planet. Sci. Lett., 70:164-168, October 1984. doi: 10.1016/0012821X(84)90002-5. 